\title{
Multiple ART Programs Create a Dilemma for Providers to Monitor ARV Adherence in Uganda
}

\author{
Celestino Obua ${ }^{*}{ }^{, 1}$, Annelie Gusdal ${ }^{2}$, Paul Waako ${ }^{1}$, John C Chalker ${ }^{3}$, Goran Tomson ${ }^{2}$, \\ Rolf Wahlström ${ }^{2}$ and The INRUD-IAA Team ${ }^{4}$
}

\author{
${ }^{I}$ Makerere University, College of Health Sciences, School of Biomedical Sciences, Department of Pharmacology and \\ Therapeutics, Kampala, Uganda \\ ${ }^{2}$ Karolinska Institutet, Department of Public Health Sciences, Division of Global Health (IHCAR), Nobels väg 9, 17177 \\ Stockholm, Sweden \\ ${ }^{3}$ Center for Pharmaceutical Management, Management Sciences for Health, Arlington, VA, USA \\ ${ }^{4}$ The International Network for the Rational Use of Drugs Initiative on Adherence to Antiretrovirals
}

\begin{abstract}
Background: Increased availability and accessibility of antiretroviral therapy (ART) has improved the length and quality of life amongst people living with HIV/AIDS. This has changed the landscape for care from episodic to longterm care that requires more monitoring of adherence. This has led to increased demand on human resources, a major problem for most ART programs. This paper presents experiences and perspectives of providers in ART facilities, exploring the organizational factors affecting their capacity to monitor adherence to ARVs.

Methods: From an earlier survey to test adherence indicators and rank facilities as good, medium or poor adherence performances, six facilities were randomly selected, two from each rank. Observations on facility set-up, provider-patient interactions and key informant interviews were carried out. The strengths, weaknesses, opportunities and threats identified by health workers as facilitators or barriers to their capacity to monitor adherence to ARVs were explored during group discussions.

Results: Findings show that the performance levels of the facilities were characterized by four different organizational ART programs operating in Uganda, with apparent lack of integration and coordination at the facilities. Of the six facilities studied, the two high adherence performing facilities were Non-Governmental Organization (NGO) programs, while facilities with dual organizational programs (Governmental/NGO) performed poorly. Working conditions, record keeping and the duality of programs underscored the providers' capacity to monitor adherence. Overall $70 \%$ of the observed provider-patient interactions were conducted in environments that ensured privacy of the patient. The mean performance for record keeping was $79 \%$ and $50 \%$ in the high and low performing facilities respectively. Providers often found it difficult to monitor adherence due to the conflicting demands from the different organizational ART programs.

Conclusion: Organizational duality at facilities is a major factor in poor adherence monitoring. The different ART programs in Uganda need to be coordinated and integrated into a single well resourced program to improve ART services and adherence monitoring. The focus on long-term care of patients on ART requires that the limitations to providers' capacity for monitoring adherence become central during the planning and implementation of ART programs.
\end{abstract}

Keywords: HIV/AIDS, antiretroviral therapy, providers, adherence monitoring, Uganda.

\section{BACKGROUND}

The rapid scale-up of antiretroviral therapy (ART) in sub-Saharan African (SSA) countries has not only increased availability and accessibility of these drugs but has also improved the length and quality of life amongst people living with HIV/AIDS (PLWHA) [1-3]. As patients on ART live longer, the landscape for care tends to change from acute or episodic care to one that now requires chronic or longterm care, and careful monitoring of adherence becomes

*Address correspondence to this author at the Department of Pharmacology and Therapeutics, School of Biomedical Sciences, College of Health Sciences, Makerere University, P. O. Box 7072, Kampala, Uganda; Tel: +256 414532 945; Fax: +256 414532 947; E-mail: cobua@chs.mak.ac.ug especially important since the level of adherence contributes to treatment outcomes [4,5]. For ART programs in SSA, monitoring of adherence is one of the most challenging activities [6], because of the underlying weaknesses in the health infrastructure, increased demand for financial and human resources, and especially the apparent decline in human resource in the public sector [7-9]. The support of adherence monitoring in SSA thus calls for effective and efficient coordination of ART programs that have strong linkages between policy, organizations and the human resource which should reflect the local magnitude and trends of HIV/AIDS.

Recent reports show that the prevalence of HIV in Uganda is on average 5.4\% [10], with about 286 facilities in 
both public and private sector providing ART to some 120,000 patients of the nearly 400,000 who were in need of therapy by 2007 [11]. These figures are likely to go upwards as the drive is to expand the provision of ARVs to lower level health facilities. It is likely that the available human resource will face challenges in coping with the burden of this expansion, especially to monitor adherence and assure the quality of care $[12,13]$.

The scale-up of ART in Uganda and other SSA countries has been successful largely because of massive efforts from international philanthropists and partners such as the GLOBAL FUND for AIDS, TB and Malaria (GFATM), the Presidential Emergency Plan for AIDS Relief (PEPFAR) and many local players from the public and private sectors. The one area that seems to have been inadequately addressed by these efforts is human resources [14]. Despite the concerted training of more health providers in HIV/AIDS clinical care provision, most public organizations have not been able to recruit and retain sufficient health workers, thus calling for innovations such as task shifting in order to cope with human resource demands. Task shifting in clinical care is the transfer or delegation of defined responsibilities to other providers usually from higher to lower cadres and sometimes to non-professionals $[15,16]$. Uganda is one of the SSA countries that has adopted task shifting as a means to mitigate deficiencies in human resource in the roll-out of ART programs, however task shifting is not without challenges for the organization as well as the health providers [10]. Experiences and perspectives of providers involved in these programs open opportunities to explore organizational factors from where new strategies could be formed [17], for effective ART adherence monitoring.

It is known that low adherence and low retention of patients on ARVs are important predictors of poor treatment outcomes [18] and the extent to which organizational factors are responsible for these are important if interventions are to be planned. For the programs, the limited numbers of health workers hinder the capacity to monitor the adherence to ART, especially where health workers are not involved in policy making and only viewed as inputs necessary for effective ART programs [19]. From the providers' view point, these challenges can only be better understood when their perspectives regarding monitoring of adherence in ART programs are explored in the light of their day-to-day experiences, such as working in multiple ART programs [20]. This paper presents experiences and perspectives of providers in ART facilities, exploring the organizational factors affecting their capacity to monitor adherence to ARVs.

\section{METHODS}

\section{Study Sites and Study Design}

This was a cross-sectional determinants study following an adherence indicators survey conducted in 20 facilities in both rural and urban settings of Uganda to determine the facility adherence performances, from which facilities were ranked either as high, medium or low adherence performance [21]. Three main adherence measures were considered before ranking; percentage of days covered by dispensed drugs over a 183-day period; average self reported adherence; and attendance to next appointment. The rankings were then based on overall mean score for all adherence and defaulting measures that scored from 1(high), 2(medium) to 3 (poor). In the original survey, four types of organizational systems were found operating in the facilities, distributed as shown in Table 1 with facility ranking. The survey showed that all facilities with mixed programs had poor adherence performance, and all purely Non-Governmental Organization/Not-For-Profit Organizations (NGO/NFPO) had high adherence performances, while most purely government program facilities had better (medium) adherence performance than the mixed programs (Table 1).

In the current study, six facilities, two from each performance level were randomly selected for in-depth study of organizational/provider determinants of monitoring adherence to ART, including governmental and nongovernmental facilities situated in rural and urban settings of the country. After randomization, the high performing facilities selected were NGO only programs, the medium performing facilities were Government (GOV) only programs, and the poor performing facilities had mix programs.

Table 1. Number of Facilities Distributed on Organizational Type and Adherence Performance Ranking from ART Facility Survey in Uganda

\begin{tabular}{|c|c|c|c|c|}
\hline \multirow{2}{*}{$\begin{array}{c}\text { Facility } \\
\text { Type/Level }\end{array}$} & \multirow{2}{*}{$\begin{array}{c}\text { Organizational } \\
\text { Type/Program }\end{array}$} & \multicolumn{2}{|c|}{ Adherence Performance Ranking } \\
\cline { 3 - 5 } & & Low & Medium & High \\
\hline \hline \multirow{2}{*}{ RRH (n=5) } & GOV & - & 2 & - \\
\cline { 2 - 5 } & Mix & 3 & - & - \\
\hline \multirow{2}{*}{ DH (n=7) } & GOV & 2 & 4 & - \\
\cline { 2 - 5 } & Mix & 1 & - & - \\
\hline NGO (n=5) & NFPO & - & - & 5 \\
\hline PVT (n=2) & FPO & - & 1 & 1 \\
\hline \multicolumn{2}{|c|}{ Total } & $\mathbf{6}$ & $\mathbf{7}$ & $\mathbf{6}$ \\
\hline
\end{tabular}

GOV-Government, NGO - nongovernmental, RRH-Regional Referral Hospital, DH-District Hospital, PVT-Private, NFPO-not for profit organization, FPO-for profit organization, Mix- GOV/NFPO.

\section{Study Population and Tools}

Staff and in-charge managers at the selected facilities were interviewed. Sit-in observations of providers' interaction with patients aged 18 years or above who were on ART for at least six months were selected conveniently as they came for care. The data collection tools consisted of incharge and staff interview, facility and sit-in observation guides. These tools were designed as checklists with the focus on identifying strengths and weaknesses that have a bearing on adherence within the following areas in the facility: organizational structure, staffing, drug supply and dispensing, daily work load, laboratory resources and integration of services in the clinic. Interviews with incharge managers were performed in order to understand the 
work procedures and the dynamics of the ART program in the facilities.

Sit-in observations were performed to observe the patient-provider interactions. A checklist was used to gather information on record keeping and content of information provided to patients; including details of physical examination, opportunistic infections (OIs) screening, adverse drug reactions (ADR), adherence monitoring and prescribing routines. The length of consultation and dispensing time were also noted. The sit-in observations provided an objective opportunity to observe the interaction in the patient-provider encounters, noting length of consultation, and if the environment was appropriate, correct information was given and whether certain encounter details were recorded. For this study, consultation time was defined as the duration from the moment the patient sat down for interactions with the provider until the patient was dismissed by the provider, while the dispensing time was defined as the time from when the dispenser called the patient to the dispensing window to be instructed and handed over their medicines to when the patient leaves the window.

In the interviews, semi-structured questionnaires were used, including open-ended questions with suggested areas for probing. Information from the perspectives of the provider on the healthcare system, the role of the patient, staff, and community in supporting adherence and beliefs on both challenging and enabling factors were addressed. In addition, the impact of the healthcare systems and provider experiences were explored using strengths, weaknesses, opportunities and threats (SWOT) forms, that were used to identify each facility's internal factors (the facility's resources and capabilities) and external factors (circumstances in which the facility operates) in providing and monitoring adherence to ART.

\section{Ethical Approval}

Ethical approval for this study was given by the Makerere University Faculty of Medicine Research and Ethics Committee. Permission to conduct the study was granted by the Uganda National Council of Science and Technology and the Uganda National AIDS Control Program. All health providers in the facilities studied were informed of the study and those who were observed gave written consent. The patients were informed and gave group verbal consent for the observations of the interactions.

\section{Data Collection and Analysis}

In each facility, during the first day, SWOT forms were given to all staff by members of the study team. Providers were asked to identify the strengths, weaknesses, opportunities and threats to high quality service regarding ART adherence in their facility and note their reflections in the SWOT forms and return them to the team on the next day. This was then followed by SWOT group discussion with $6-8$ staff members. The team leader presented an overview of the facility findings, the sit-in observations, and a summary of the SWOT materials filled in by staff. A discussion followed on the factors affecting monitoring of adherence at the facilities, including the possible and feasible interventions or other remedial actions to overcome deficiencies in adherence performance. At all facilities, interviews and SWOT discussions were conducted in English and were audio recorded. The analysis of the qualitative data followed an inductive approach. Audio recordings were transcribed verbatim. These were then read thoroughly and minutely to allow conceptual clusters of ideas and patterns to emerge, which were manually organized into descriptive content areas with sublevels. For quantitative data, descriptive analysis was performed using SPSS version 12, and the results presented as frequencies or tabulated.

\section{RESULTS}

\section{Sit-In Observations of Patient-Provider Interactions}

A total of 96 provider-patient interactions were observed, ranging from 14 to 21 per facility. Staff numbers were higher in the higher performing facilities than in the lower facilities, while the number of patients attending on a clinic day ranged from 31 to 267 (Table 2). The providers included doctors, clinical officers and nurses among the prescribing team with higher staff to patient ratios in the higher performing facilities than in the lower performing ones. More than $2 / 3$ $(70 \%)$ of the patient-provider interactions were conducted in environments that ensured patient privacy within the facilities. The high performing facilities had better consultation environment compared to the low performing facilities. Most (76\%) encounters lasted on average under 10 minutes, with $19 \%$ between $10-15$ minutes, and only $5 \%$ lasting more than 15 minutes. There was a significant difference in mean consultation time between the high and the lower performing facilities $(8.2 \pm 2.7$ vs $6.4 \pm 2.4$, t-test $\mathrm{p}=0.001$ at $95 \%$ CI $0.7-2.8)$. Most facilities had similar dispensing time except for one of the high adherence facilities that had nearly double length of dispensing time (Table 2), however comparison of the overall mean dispensing time between the high and lower performing facilities $(66.0 \pm 24.6$ vs 31.2 \pm 8.6$)$ were significant (t-test $\mathrm{p}<0.001$ at $95 \%$ CI $39.5-49.0)$.

\section{Information Provision to Patients}

Overall most encounters showed insufficiencies in the information given to the patients about HIV and ART regarding the importance of adherence, secondary prevention and about adverse drug reactions (ADRs) and OIs. It was further observed that provision of information in the areas studied was not clearly associated with facility performance in terms of ART adherence as the poor performance clinics provided information more often than the high performing clinics (Table 3).

\section{Interviews and Group Discussions}

From the analysis of the interviews and SWOT discussions, three main themes emerged related to the impact of healthcare systems on the capacity of providers to monitor adherence to ARVS The first theme describes providers' unstable working conditions; the second theme adresses the important role of records keeping in adherence monitoring 
Table 2. Facility Observation on Staff, Provider-Patient Interactions and Other Functionality Parameters

\begin{tabular}{|c|c|c|c|c|c|c|c|}
\hline \multicolumn{2}{|c|}{ Observed Characteristics } & \multicolumn{2}{|c|}{ High Adherence } & \multicolumn{2}{|c|}{ Medium Adherence } & \multicolumn{2}{|c|}{ Low Adherence } \\
\hline & & $\begin{array}{l}\text { Facility1 } \\
\text { (NGO) }\end{array}$ & $\begin{array}{c}\text { Facility2 } \\
\text { (NGO) }\end{array}$ & $\begin{array}{c}\text { Facility } 1 \\
\text { (GOV) }\end{array}$ & $\begin{array}{c}\text { Facility2 } \\
\text { (GOV) }\end{array}$ & $\begin{array}{c}\text { Facility1 } \\
\text { (Mix) }\end{array}$ & $\begin{array}{c}\text { Facility2 } \\
\text { (Mix) }\end{array}$ \\
\hline \multirow[t]{7}{*}{ Staffing Situation } & Doctors & 6 & 1 & 1 & 0 & 0 & 2 \\
\hline & Clinic Officer & 0 & 1 & 1 & 1 & 3 & 1 \\
\hline & Nurses & 9 & 2 & 1 & 1 & 1 & 5 \\
\hline & Counsellors/community & 21 & 4 & 2 & 0 & 1 & 0 \\
\hline & Pharmacist/Technicians & 3 & 3 & 0 & 0 & 0 & 4 \\
\hline & Data Clerks & 2 & 1 & 0 & 0 & 0 & 1 \\
\hline & Total staff at clinic & 41 & 12 & 5 & 2 & 5 & 13 \\
\hline \multicolumn{2}{|c|}{ Number of patients attending per visit day } & 201 & 32 & 102 & 31 & 267 & 249 \\
\hline \multicolumn{2}{|c|}{ Staff per patient visit } & 0.20 & 0.37 & 0.05 & 0.06 & 0.02 & 0.05 \\
\hline \multicolumn{2}{|c|}{ Number of Provider-patient interactions observed } & 15 & 19 & 16 & 16 & 15 & 15 \\
\hline \multicolumn{2}{|c|}{ Consultation time (average in minutes) } & 8 & 9 & 6 & 5 & 6 & 7 \\
\hline \multicolumn{2}{|c|}{ Dispensing time (average in seconds) ${ }^{\mathrm{a}}$} & 41 & 90 & 25 & 30 & 25 & 45 \\
\hline \multicolumn{2}{|c|}{ Laboratory functionality } & Full & Full & Partial & Partial & Partial & Partial \\
\hline \multicolumn{2}{|c|}{ Number of opening days per week } & 5 & 5 & 1 & 1 & 3 & 5 \\
\hline
\end{tabular}

${ }^{\text {a }}$ Dispenser-patient interaction time from when the patient is called to the dispensing counter to receive medicines to the time he leaves the counter.

Table 3. Information Provided to Revisiting Patients from Sit-In Observations

\begin{tabular}{|l|c|c|c|c|}
\hline \multirow{2}{*}{\multicolumn{1}{c|}{ Type of Information Provided }} & \multicolumn{3}{c|}{ Number (\%) of Consultations by Facility Ranking } \\
\cline { 2 - 5 } & $\begin{array}{c}\text { Overall } \\
\mathbf{n}=\mathbf{9 6}\end{array}$ & $\begin{array}{c}\text { High } \\
\mathbf{n}=\mathbf{3 4}\end{array}$ & $\begin{array}{c}\text { Medium } \\
\mathbf{n}=\mathbf{3 2}\end{array}$ & $\begin{array}{c}\text { Low } \\
\mathbf{n}=\mathbf{3 0}\end{array}$ \\
\hline \hline General HIV information & $4(4)$ & $1(3)$ & $3(0)$ & $0(0)$ \\
\hline Information on specific ART & $5(5)$ & $1(3)$ & $5(17)$ \\
\hline Information on importance of adherence & $28(29)$ & $15(44)$ & $5(16)$ & $8(27)$ \\
\hline Information on secondary prevention & $27(28)$ & $13(38)$ & $7(22)$ & $7(23)$ \\
\hline Information about the prescribed drugs & $9(9)$ & $3(9)$ & $7(0)$ & $6(20)$ \\
\hline Enquiry about adverse drug reactions & $21(22)$ & $7(21)$ & $5(16)$ & $9(30)$ \\
\hline Enquiry about opportunistic infections ${ }^{\mathbf{a}}$ & $58(60)$ & $29(85)$ & $18(56)$ & $11(37)$ \\
\hline
\end{tabular}

${ }^{a}$ Opportunistic infections such as pnuemocystis carinii pneumonia and cryptoccocal meningitis.

and the third theme describes providers' perspectives on the quality of the health systems and duality of ART programs.

\section{Organizational Systems and Working Conditions}

Generally there were inadequate and/or unstable staffing situations in the facilities studied. In the case of high performing facilities this was due to the high turnover of staff and inability to retain them even though the facilities are able to recruit more staff. In the case of lower performing facilities, this was often due to a lack of dedicated staff for the ART clinics, as the clinics shared staff with other nonART provision units. There were prolonged waiting times, patient congestion, and an inability to carry out activities and record parameters necessary to monitor adherence. In facilities where more than one system was managing ARV provision (government and NGO) it was found that the provider often encountered difficulties in fulfilling the obligations of the different systems, such as the different formats of records required, ARV enrolment criteria, splitting patients in the different program regimens, and the different follow-up or patient tracking systems. Although facilities were open for as few as one day a week (in the low performing facilities) to five days a week (in the high performing facilities) as shown in Table $\mathbf{3}$, it is difficult to test any correlation between the opening days with the adherence performance of the facilities due to the few number of facilities in the study.

At facility level some of the major program challenges to effective adherence monitoring included: 
- Poor/lack of laboratory service, especially the inability to perform CD4 tests was seen as a major hindrance to effective monitoring of adherence.

- $\quad$ Lack of OI drugs - frequent stock-outs of OI drugs increases the cost of adherence as patients are forced return to the clinics many times.

- Few staff and heavy patient load, inadequate remuneration and high staff turnover all negatively influence the quality services including adherence counselling and monitoring.

All facilities were severely understaffed and the attrition rate was particularly high among doctors, where flexibility in function, rotation of staff between different departments, and task shifting was used as the main tool to bridge the human resource gaps in the facilities.

Given the limited numbers of doctors, many of our nurses here are prescribing medicines and they are doing it very well [In-charge manager, $\mathrm{NGO}$.

However, task shifting often constrained the already overworked providers and, together with negative perceptions of the ART unit, providers were often unwilling to fulfil the tasks they had been assigned. The availability of providers to meet patients' demands was however critical. Providers were seriously concerned about the current patient load and the increase in the number of patients, which could not be stopped, while the number of health care providers remained the same or even decreased affecting their ability to monitor adherence.

\section{...because of the workload when it gets to around 2:00 pm people who started by singing adherence in a vibrant manner start losing interest to talk about the same because they are already worn out; and in the long run we lose the touch of adherence. [In-charge manager, GOV/NGO].}

Furthermore, the role of providers went beyond providing medications only. Providers also acted as educators and trainers of professional counsellors, peer counsellors, community based volunteers and the general public. They however decried their own lack of training in adherence monitoring.

We have never gone for the training and we don't know whether what we are doing is right [Clinical officer, GOV].

The flipside of training providers in adherence support was that they became more "marketable" for other more financially attractive organizations to employ them, referred to as "internal brain-drain".

.....there is a problem that the attrition rate, is too high especially when it comes to clinicians. We send them for further training. So after training of course they become more marketable and of course there are many organisations that are ready to employ them [In-charge manager, NGO].

\section{Record Keeping as an Important Issue in Adherence Monitoring}

A well-organized record keeping system distinguished the higher from the lower performing facilities. It was observed that high performing facilities had well organized patient tracking and record systems, often with trained records assistants in place. During patient-provider interactions, providers in high performing facilities recorded the date of the visit, the date of next the visit, the drugs and the number of medication days prescribed more frequently compared to the low performing. The mean performance for recording was significantly better in the high performing $(78.7 \pm 39.1 \%)$ compared to the low performing facilities $(50.3 \pm 42.4 \%)$ with $\mathrm{t}$-test for equality of means giving $\mathrm{p}=0.01$ at $95 \% \mathrm{CI}$ of $6.89-50.05$. Information regarding the patients' current symptoms, laboratory examination results, physical examinations and results of clinical examinations were often not recorded in lower performing facilities. Only having an attendance record, often taken by volunteers, did not indicate what the person had come to do at the clinic.

...but you see, when you have semi-skilled
manpower you have to talk over this issue
(records keeping) with a lot of push ......... So
by that I say that the semi-skilled people
(volunteers) sometimes don't know the
importance of what you wanted. Yet if you
talked to somebody who understands what
data implies it would certainly be easy [In-
charge manager, GOV/NGO].

In all facilities the tool frequently used to capture patient record was the "blue card", the patients" ART comprehensive care card, considered to capture most data important for care and adherence monitoring. In lower performing facilities missing records were often noticed. In facilities with dual programs, proper record keeping was poor due to the complexity of the different record systems that the providers had to cope with, and this was further compounded by provider shortage.

\section{Because of the poor staffing, poor recording goes on.... to explain why at times certain things go on, because you are the same person doing this and doing the other, some things may skip your mind [In-charge manager, GOV/NGO].}

\section{Providers' Perspectives on the Organisation and Duality of ART Programs}

The interviews and discussions paint a picture of many serious constraints, sometimes made worse by the fact that multiple programs ran concurrently. The providers believed there was no integration or coordination of the various programs they implemented in the facilities. Even the drugs were differently sourced. Running parallel NGO/NFPO and Government funded programs within the same facilities presented system conflicts. These dual programs proved disadvantageous to providers and often portraying providers 
negatively in the eyes of patients as providers have to select patients to fit into the different programs.

Of all the barriers identified by the providers, drug shortages and stock outs were the most frustrating. Providers advised their patients to take medications daily for life without missing out on doses but when patients came to collect their medication there may be none to offer. The situation is more complex where drugs are available for one program (NGO) and lacking for the other program (GOV) in a facility with dual programs. Failure to provide medications due to stock out greatly complicated adherence monitoring.

Sometimes, we have drug stock outs. This is a big problem and a big challenge affecting the clinic. We tell the patients that once you begin on drugs you take them for life yet they come and we tell them that we have no drugs. This is to them almost sending them to go and die. We don't know what to do... [Clinical officer, GOV].

Recurring shortages of ARVs and OI drugs often lead to borrowing/lending of drugs, substitution of regimens, and sometimes giving drugs for shorter intervals, say for 2 weeks, even to "stable" patients who normally would have returned to the clinic after one month or longer. In the facilities with dual programs, providers explained that the reasons for the short supplies were partly due to the donors' restrictions on the number of patients to treat and yet more patients may qualify for therapy in the Gov program. The other reasons were usually miscommunication and bottlenecks in supply logistic as drugs were distributed from several different donors and procurement chains.

...we used to make requisition but then we found there were no drugs in stock. I think there was a problem somewhere. The other time they (Ministry of Health) said the drugs have come but they had not yet. Something had not been done so they could not give them to us. .like there was a logistic problem somewhere [In-charge Manager, GOV].

\section{Laboratories in Adherence Monitoring}

Providers viewed the functionality of laboratories as important to proper adherence monitoring within the programs. While facilities had laboratory technicians, these were trained only on how to use the equipments but not on maintenance or servicing. The facilities most often did not know whom to report laboratory difficulties to, and confusion regarding the responsibility for the services was evident. None the six facilities performed viral load tests, while CD4 testing were only performed in NGO/NFPO facilities, and in mixed facilities this was only offered to patients enrolled on the NGO programs, making the services and monitoring adherence at these facilities inadequate. Providers at all government facilities had to rely on their clinical assessment in the initiation of therapy and adherence monitoring.

The lab ....can't do for me the viral load, which we are interested in, they can't do for me a CD4 count, I mean these electronic tests. There are things like the renal function test and liver function test. Those ones I just forget about them. Blood chemistry cannot be done, yeah, which is very important. .. we can't do it. So we ask for a hand from somewhere else [Incharge manager, GOV/NGO].

\section{DISCUSSION}

In exploring the functionality of organizational systems in ART facilities and the perspectives of health workers regarding adherence monitoring in ART programs in Uganda, it emerged that both internal and external factors were responsible for the level of adherence monitoring. The organizational systems operating in various facilities majorly contributed to these factors. In monitoring of adherence, the high performing facilities were all found to be purely NGO/NFPO, while the purely GOV programs had medium performance and the poor performing facilities had mixed (dual) GOV/NGO programs. This indicated that whenever there was a mix in the organizational systems at the facilities, adherence performances were not optimal. The entry of NGO programs into HIV/AIDS care and service improved access to ARVs low income countries such as Uganda. A study that looked at the coordination of health sector aid in Zambia showed that while aid support to HIV/AIDS increased, integration of partners at delivery points was lacking [22]. The health workers are then presented with difficulties in carrying out routine activities such as having to make decisions on which patients to recruit in a program, how to keep dual record systems, let alone monitor the patients as often staffing levels do not change [13].

Where there was duality of programs at a facility, it was found that the capacity of health providers to perform optimally was adversely affected. This was associated with poor rankings of such facilities. The multiplicity of requirements in the dual programs challenges the providers with the dilemma of fulfilling the competing demands. While the NGO programs may have been introduced at the GOV facilities to support the government efforts in scaling up the delivery of ARVs, it is possible that the provider capacity to handle the dual programs may not have been taken into account [19]. Secondly the lack of coordination and systematic integration at delivery points has also proved to be an important weakness in these mixed programs [22]. A study that compared three different delivery systems at facility level in Uganda also found that lack of collaboration between the systems was the main challenge to effective ART delivery in the programs [20]. In the present study, providers generally found it difficult to perform the different tasks simultaneously, and at times this was simply impossible. It is therefore imperative that the dual programs undergo complete integration so that they are presented at the facilities as one program, making it easy for the few staff at the facilities to deliver services such as giving longer consultation time, providing better information, counselling and record keeping, which are key components for adherence monitoring [23]. 
Health providers are important partners in the health systems or organizations [19]. As such, their perspectives on what is important in the implementation of the programs at the facility level should be understood and addressed. Each provider can only do so much at a time, hence even when they are expected to diversify roles during task shifting the attendant limitations should be recognized. To ensure quality services, a systematic and well targeted approach to task shifting is a requirement [15]. In this study, task shifting was found to be spontaneous in response to the lack of clinic dedicated staff, as this only served to bridge the immediate man-power gaps, hence performance did not necessarily improve. This is perhaps why GOV and the mixed program facilities in the present study were found to have performed less than optimally despite the task shifting [12,13].

Staff training is important for performance improvement, but numbers are also important. Providers indicated that while they would like specific training, more staff should be employed and these should be dedicated to ART clinics. Where this occurred, especially in NGO program facilities, performance in adherence monitoring was often better. In most GOV and Mixed programs, lack of trained dedicated ART clinic staff and inability to adequately interact with patients was perceived as contributing to their incapacity to monitor adherence optimally. Similarly, in a meta-analysis that compared adherence to ART in SSA and North America, it was found that monitoring adherence was inadequate due to multiple program factors especially poor provider interaction with patients [24].

While adherence to ART is often the responsibility of the patient [25], the role of providers in ensuring this often goes beyond providing medications only. In the present study, providers performed other functions including being educators to patients and other staff. If monitoring of adherence is to be carried out optimally, program planning must therefore include provision for adequate staff recruitment, retraining and retention. In this study, providers agreed that their performance in monitoring adherence was often poor because the health system planners appeared not to have taken into account the need to recruit and retain staff at the facilities. The frequent internal brain drain occurs due to the fact the there is a proliferation of NGO programs which all require trained staff. These compete by hiking remunerations to attract the already trained staff from other programs. The apparent brain drain is not without some positive effects, as at the wider health systems level it helps to retain trained health workers within the health system in the country [26], as opposed to the external brain drain that has destabilized the SSA health systems. If NGO ART programs were integrated into the GOV programs there would be more stability in staffing and minimal staff turnover. While the many ART programs may improve the number of patients with access to ARVs, there is usually no proportional increase in health workers, and failure to take into account what it would take to monitor adherence to the medications in the long run is a disservice to the patients [27].
In this study it was observed that diagnosis and follow-up of patient progress is mainly based on clinical assessment. While this is a useful for diagnosis and assessment for initiation of therapy, most typical clinical features change once patients have been on therapy for some period. This makes clinical assessment unreliable for monitoring of adherence. The present study showed that facilities with lower adherence monitoring did not routinely use other assessment methods, such as CD4 tests, to monitor adherence and inability to perform laboratory tests in monitoring ART is a frequent limitation in most SSA ART program [28].

Providers often feel wrongly vilified for external system problems over which they have no control, such as drug and staff shortages, non-functional laboratories, and parallel programs which seem to bring discontent among the patient communities. Having to turn patients back due to lack of drugs is as painful to the providers as it is to the patients and so, they feel deflated and discouraged, hence the desire to seek for transfer to better performing organizations. The number of providers required for optimal services and adherence monitoring is projected to double yearly for the next decade [29]. This is a real dilemma that threatens the successes in scale-up of ART programs [30]. In this study the single program facilities had a four to ten times better staffing ratio, with better adherence monitoring, further emphasizing the need for optimizing the number of providers at the facilities.

However whether providers are completely free of blame for systems failures or whether reorganization of programs by centrally coordinating them into a single integrated program will indeed improve services and adherence monitoring are issues that will require a wider study that should involve policy level down to the facilities, the providers inclusive.

\section{CONCLUSIONS}

Organizational duality at facilities is a major factor in poor adherence monitoring. The different ART programs in Uganda need to be coordinated and integrated into a single well resourced program to improved ART services and adherence monitoring. The focus on long-term care of patients on ART requires that the limitations to provider capacity for monitoring adherence become central during the planning and implementation of ART programs.

\section{COMPETING INTERESTS}

The authors declare no competing interests. This work was made possible through support by the Swedish International Development Cooperation Agency, under the terms of contribution 72300310, the World Health Organization under an Agreement for Performance of Work: OD-AP-07-00516 and by the Rational Pharmaceutical Management Plus Program, which is funded by the U.S. Agency for International Development, under the terms of Cooperative Agreement number HRN-A-00-00-00016-00. The opinions expressed herein are those of the author(s) and do not necessarily reflect the views of the Swedish International Development Cooperation Agency, the World 
Health Organization or the U.S. Agency for International Development.

\section{AUTHORS' CONTRIBUTIONS}

$\mathrm{RW}, \mathrm{GT}$ and JC designed and planned the research, designed the questionnaires and interview guides, analyzed the data. CO, AG and PW commented on the design of the questionnaires and the interview guides, selected facilities to survey, carried out the survey and assisted in the analysis of the data. $\mathrm{CO}$ drafted the article. All authors commented on article drafts and approved the final version.

\section{ACKNOWLEDGEMENTS}

The authors would like to acknowledge the other members of the INRUD Initiative on Adherence to Antiretrovirals, which include staff at the national AIDS control programs in Uganda, INRUD-Uganda, and Management Sciences for Health country office in Uganda, as well as staff in partner organizations: Harvard Medical School and Harvard Pilgrim Health Care Institute, Boston, USA; Division of Global Health IHCAR, the Karolinska Institutet, Stockholm, Sweden; Center for Pharmaceutical Management, Management Sciences for Health, Arlington, USA; and Department of Essential Medicines and Pharmaceutical Policies, World Health Organization, Geneva, Switzerland.

\section{REFERENCES}

[1] Bangsberg DR, Perry S, Charlebois ED, et al. Non-adherence to highly active antiretroviral therapy predicts progression to AIDS. AIDS 2001; 15: 1181.

[2] Garcia DEO. Impact of adherence and highly active antiretroviral therapy on survival in HIV-infected patients. J Acquir Immune Defic Syndr 2002; 30: 105-10.

[3] World Health Organization, UNAIDS. Progress on global access to HIV antiretroviral therapy: a report on " 3 by 5 " and beyond. WHO 2006.

[4] Frick PA, Gal P, Lane TW, Sewell PC. Antiretroviral medication compliance in patients with AIDS. AIDS Patient Care STDS 1998; 12: $463-70$.

[5] Swendeman D, Ingram BL, Rotheram-Borus MJ. Common elements in self-management of HIV and other chronic illnesses: an integrative framework. AIDS Care 2009; 21: 1321-34.

[6] Chalker J, Andualem T, Minzi O, et al. Monitoring adherence and defaulting for antiretroviral therapy in 5 east African countries: an urgent need for standards. J Int Assoc Physicians AIDS Care 2008; 7: 193.

[7] Assefa $\mathrm{Y}, \mathrm{Kloos} \mathrm{H}$. The public health approach to antiretroviral treatment (ART) service scale-up in Ethiopia: the first two years of free ART, 2005-2007. Ethiop Med J 2008; 46: 401.

[8] Janse RBE, Engelbrecht M, Steyn F, et al. Resources and infrastructure for the delivery of antiretroviral therapy at primary health care facilities in the Free State Province, South Africa. SAHARA J 2008; 5: 106.

[9] World Health Organization. Working together for health. In; 2006. www.who.int/whr/2006/whr06_en.pdf [Accessed 10th Dec 2009].

[10] UNFPA. State of World Population 2009. Facing a changing world: Women, population and Climate. In; 2009, www.unfpa.org/ swp/2009/en/ [Accessed, 10 ${ }^{\text {th }}$ Dec 2009].
[11] World Health Organization, UNAIDS, UNICEF. Epidemiological Fact Sheets on HIV and AIDS, 2008 Update. In; 2008, www.who. int/globalatlas/.../EFS2008/full/EFS2008_HT.pdf [Accessed，9th Dec 2009].

[12] Philips M, Zachariah R, Venis S. Task shifting for antiretroviral treatment delivery in sub-Saharan Africa: not a panacea. Lancet 2008; 371: 682-4.

[13] Torpey KE, Kabaso ME, Mutale LN, et al. Adherence support workers: a way to address human resource constraints in antiretroviral treatment programs in the public health setting in Zambia. PLoS One 2008; 3: 2204

[14] Kober K, Van Damme W. Scaling up access to antiretroviral treatment in southern Africa: who will do the job? Lancet 2004; 364: 103-7.

[15] Morris MB, Chapula BT, Chi BH, et al. Use of task-shifting to rapidly scale-up HIV treatment services: experiences from Lusaka, Zambia. BMC Health Serv Res 2009; 9: 5.

[16] Zachariah R, Ford N, Philips M. Task shifting in HIV/AIDS: opportunities, challenges and proposed actions for sub-Saharan Africa. Trans R Soc Trop Med Hyg 2009; 103: 549-58.

[17] Hermann K, Van Damme W, Pariyo GW, et al. Community health workers for ART in sub-Saharan Africa: learning from experiencecapitalizing on new opportunities. Hum Resour Health 2009; 7: 31.

[18] World Health Organization. Towards universal access: scaling up priority HIV/AIDS interventions in the health sector. In; 2009, http://www.who.int/hiv/pub/2009progressreport/en/ [Accessed 15 $5^{\text {th }}$ Jan 2010].

[19] Rajaraman D, Palmer N. Changing roles and responses of health care workers in HIV treatment and care. Trop Med Int Health 2008 13: 1357-63.

[20] Larsson EC, Okong P, Thorson A, Ekström AM. Antiretroviral treatment of HIV in Uganda: a comparison of three different delivery models in a single hospital. Trans R Soc Trop Med Hyg 2007; 101: 885-92.

[21] Chalker J, Andualem T, Gitau L, et al. Measuring adherence to antiretroviral treatment in resource-poor settings: The feasibility of collecting routine data for key indicators. BMC Health Serv Res 2010; $10: 43$.

[22] Sundewall J, Forsberg BC, Jönsson K, Chansa C, Tomson G. The Paris Declaration in practice: challenges of health sector aid coordination at the district level in Zambia. Health Res Policy Syst 2009; 7: 14.

[23] World Health Organization. Patient monitoring guidelines for HIV care and antiretroviral therapy (ART). In; 2006, www.who.int/ 3by5/capacity/ptmonguidelinesfinalv1.PDF [Accessed 7th Feb 2010].

[24] Mills EJ, Nachega JB, Buchan I, et al. Adherence to antiretroviral therapy in sub-Saharan Africa and North America: a meta-analysis. JAMA 2006; 296: 679 .

[25] Ware NC, Idoko J, Kaaya S, et al. Explaining adherence success in sub-Saharan Africa: an ethnographic study. PLoS Med 2009; 6: e11.

[26] Assefa Y, Jerene D, Lulseged S, Ooms G, Van Damme W. Rapid scale-up of antiretroviral treatment in Ethiopia: successes and system-wide effects. PLoS Med 2009; 6: e1000056.

[27] Sanjobo N, Frich JC, Fretheim A. Barriers and facilitators to patients'adherence to antiretroviral treatment in Zambia: a qualitative study. SAHARA J 2009; 5: 136.

[28] Landman KZ, Kinabo GD, Schimana W, et al. Capacity of healthcare facilities to deliver HIV treatment and care services, Northern Tanzania, 2004. Int J STD AIDS 2006; 17: 459.

[29] Bärnighausen T, Bloom DE, Humair S. Human resources for treating HIV/AIDS: needs, capacities, and gaps. AIDS Patient Care STDS 2007; 21: 799-812.

[30] Marchal B, Brouwere VD, Kegels G. Viewpoint: HIV/AIDS and the health workforce crisis: What are the next steps? Trop Med Int Health 2005; 10: 300-4. 